\title{
A review of tazarotene in the treatment of photodamaged skin
}

\author{
Stephanie Ogden' \\ Miny Samuel ${ }^{2}$ \\ Christopher EM Griffiths' \\ 'Dermatology Centre, University of \\ Manchester, Hope Hospital, Salford \\ Manchester, M6 8HD, UK; ${ }^{2}$ RTI- \\ Health Solutions, Williams House, \\ Manchester Science Park, Lloyd Street \\ North, Manchester MI 5 6SE, UK
}

Correspondence: S Ogden

Dermatology Centre, Irving Building, Hope Hospital, Salford, M6 8HD, UK

Tel +44 I6I 2064392

Fax +44 I6I 206 I095

Email stephanie.ogden@hotmail.co.uk

\begin{abstract}
Chronic sun exposure leads to photodamage, which is characterized clinically by fine and coarse wrinkles, dyspigmentation, telangiectasia, laxity, roughness and a sallow appearance. Many treatments claim to reduce the signs of photodamage, however evidence from randomized controlled trials (RCT) to support these claims is limited. The use of topical retinoids, particularly tretinoin, isotretinoin and tazarotene, has been shown to significantly reduce signs of photodamage both clinically and histologically. Over recent years a number of RCTs, have affirmed that topical tazarotene is an effective and safe treatment for photodamaged skin.
\end{abstract}

Keywords: photodamage, photoaging, tazarotene, retinoids

Chronic or repeated exposure to sunlight light leads to photodamage, which is manifest clinically by fine and coarse wrinkles, increased tactile roughness, laxity, dyspigmentation, sallowness and telangiectasia (Yaar and Gilchrest 1990). Histological changes include epidermal atrophy, increased melanogenesis, degeneration and disorganization of dermal collagen and fibrillin and deposition of abnormal elastic tissue (Hadshiew et al 2000; El-Domyati et al 2002)

Topical retinoids are one of the few interventions to have RCT evidence for ability to reduce clinical and histological signs of photodamage: for example regular application of topical tretinoin can reduce fine wrinkles, dyspigmentation and tactile roughness, accompanied by histological evidence of repair of dermal extracellular matrix (Kang, Bergfield et al 2005; Samuel et al 2005). Based on available evidence the US Food and Drug Administration have approved $0.05 \%$ and $0.02 \%$ tretinoin emollient creams, and $0.1 \%$ tazarotene cream for the treatment of photodamage (Kang et al 2005).

Retinoids can affect gene transcription both directly and indirectly for example by acting directly on genes that contain retinoid response elements, or indirectly by inhibiting transcription. They induce their effects mainly through binding to and activation of two families of nuclear retinoid receptors: retinoic acid receptor (RAR) and retinoid X receptor (RXR). Each family has 3 receptor subtypes: $\alpha, \beta$, and $\gamma$. Receptor selective retinoids have been developed with the aim of maximal efficacy with minimal adverse effects. Tazarotene is a synthetic receptor selective retinoid, whose active metabolite, tazarotenic acid, binds selectively to RAR $\beta$ and $\gamma$ (Chandraratna 1996).

This review article assesses the evidence for the efficacy of topical tazarotene in the treatment of photodamaged skin. Publications were identified by searching Medline, Ovid, and the Cochrane Library Databases for the terms "tazarotene", "photoaging" and "photodamage". All RCTs reporting clinically relevant outcomes were included (Table 1). 
Table I Summary of trials investigating use of topical tazarotene in the treatment of photodamaged skin

\begin{tabular}{|c|c|c|c|c|}
\hline Trial & Study design & $\mathbf{N}$ & Intervention & Main outcome measures \\
\hline $\begin{array}{l}\text { Sefton et al } \\
2000\end{array}$ & $\begin{array}{l}\text { Randomized, double- } \\
\text { blind within patient } \\
\text { comparison }\end{array}$ & 10 & $\begin{array}{l}\text { Tazarotene } 0.1 \% \text { gel applied } \\
\text { daily to one forearm, placebo } \\
\text { to opposite arm for } 12 \text { weeks }\end{array}$ & $\begin{array}{l}\text { Roughness, mottled } \\
\text { hyperpigmentation, laxity }\end{array}$ \\
\hline $\begin{array}{l}\text { Kang et al } \\
2001\end{array}$ & $\begin{array}{l}\text { Randomized, } \\
\text { investigator-masked, } \\
\text { vehicle-controlled } \\
\text { parallel comparison }\end{array}$ & 349 & $\begin{array}{l}0.01 \%, 0.025 \%, 0.05 \%, 0.1 \% \\
\text { tazarotene creams versus } \\
\text { vehicle and } 0.05 \% \text { tretinoin } \\
\text { emollient cream applied daily } \\
\text { to facial skin for } 24 \text { weeks }\end{array}$ & $\begin{array}{l}\text { OIA, global response, mottled } \\
\text { hyperpigmentation, lentigines, } \\
\text { fine and coarse wrinkles, } \\
\text { melasma, elastosis, irregular } \\
\text { dyspigmentation, tactile } \\
\text { roughness, pore size, } \\
\text { telangiectasia, actinic keratoses }\end{array}$ \\
\hline $\begin{array}{l}\text { Phillips et al } \\
2002\end{array}$ & $\begin{array}{l}\text { Randomized, double- } \\
\text { blind, vehicle- } \\
\text { controlled, parallel } \\
\text { comparison followed } \\
\text { by open-label extension }\end{array}$ & 563 & $\begin{array}{l}\text { Tazarotene } 0.1 \% \text { cream versus } \\
\text { vehicle applied daily to facial skin } \\
\text { for } 24 \text { weeks, followed by } \\
\text { tazarotene } 0.1 \% \text { cream daily for } \\
28 \text { weeks for all continuing patients }\end{array}$ & $\begin{array}{l}\text { Fine wrinkling and mottled } \\
\text { hyperpigmentation }\end{array}$ \\
\hline $\begin{array}{l}\text { Lowe et al } \\
2004\end{array}$ & $\begin{array}{l}\text { Randomized, double } \\
\text { blind, parallel } \\
\text { comparison of two } \\
\text { treatments }\end{array}$ & 173 & $\begin{array}{l}\text { Tazarotene } 0.1 \% \text { cream versus } \\
0.05 \% \text { tretinoin emollient } \\
\text { cream applied daily to facial } \\
\text { skin for } 24 \text { weeks }\end{array}$ & $\begin{array}{l}\text { Incidence of subjects achieving } \\
\geq 50 \% \text { global improvement }\end{array}$ \\
\hline $\begin{array}{l}\text { Machtinger et al } \\
2004\end{array}$ & $\begin{array}{l}\text { Randomized, double- } \\
\text { blind, vehicle-controlled } \\
\text { parallel comparison }\end{array}$ & 50 & $\begin{array}{l}\text { Tazarotene } 0.1 \% \text { cream versus } \\
\text { vehicle applied daily to facial } \\
\text { skin for } 24 \text { weeks }\end{array}$ & $\begin{array}{l}\text { Histological assessment for } \\
\text { keratinocytic and melanocytic } \\
\text { atypia }\end{array}$ \\
\hline $\begin{array}{l}\text { Kang, Krueger et al } \\
2005\end{array}$ & $\begin{array}{l}\text { Randomized, double- } \\
\text { blind, vehicle- } \\
\text { controlled }\end{array}$ & 568 & $\begin{array}{l}\text { Tazarotene } 0.1 \% \text { cream versus } \\
\text { vehicle cream applied once daily } \\
\text { to facial skin for } 24 \text { weeks }\end{array}$ & $\begin{array}{l}\text { Fine wrinkling and mottled } \\
\text { hyperpigmentation }\end{array}$ \\
\hline $\begin{array}{l}\text { Lowe et al } \\
2006\end{array}$ & $\begin{array}{l}\text { Randomized double- } \\
\text { blind comparison }\end{array}$ & $13 \mid$ & $\begin{array}{l}\text { Tazarotene } 0.1 \% \text { cream versus } \\
\text { tazarotene } 0.1 \% \text { cream plus } \\
4 \% \text { hydroquinone cream to } \\
\text { facial skin for } 24 \text { weeks }\end{array}$ & $\begin{array}{l}\text { OIA, global response, mottled } \\
\text { hyperpigmentation, lentigines, } \\
\text { fine and coarse wrinkles, } \\
\text { melasma, elastosis, irregular } \\
\text { dyspigmentation, tactile roughness }\end{array}$ \\
\hline
\end{tabular}

Abbreviations: OIA, overall integrated assessment of photodamage; $\mathrm{N}$, number.

\section{Clinical response to tazarotene}

A pilot study supported by Allergan Inc, Irvine, Ca, USA of 10 female volunteers aged between 45 and 65 years with moderately photodamaged skin, used a randomized doubleblind within patient comparison to compare the effects of tazarotene $0.1 \%$ gel applied to one photodamaged forearm versus placebo to the opposite arm once daily for 12 weeks (Sefton et al 2000). Outcome measures included changes in erythema, roughness, laxity, mottling, skin thickness and transepidermal water loss (TEWL). Skin biopsies were taken at weeks 0 and 12 from both forearms. Tazarotene gel as compared to vehicle gel resulted in significant $(p<0.05)$ improvement of skin smoothness as measured by changes in surface topography captured by silicone replicas taken at the start and the end of the 12-week study period. Although there was no detectable difference in skin thickness on ultrasound, histological analysis showed a significant increase in epidermal thickness with tazarotene treatment compared with vehicle (mean increases of $90 \%$ and $14 \%$, respectively $\mathrm{p}=0.008$ ). TEWL, hygroscopity and water holding capacity were all significantly increased by tazarotene compared with vehicle. Overall histological analysis showed at least moderate improvement in 7 of 9 participants on the tazarotene treated side only (1 subject dropped out of the study, although no reason is given for this) based on evaluation of several changes including acanthosis (thickened epidermis), hyperplastic keratinocytes, correction of keratinocyte atypia and widened intercellular spaces due to an accumulation of hyaluronic acid. All subjects experienced local side effects on the tazarotene treated forearm including erythema (all of which resolved within 2 weeks) leading to difficulty in maintaining the study blind.

In order to further investigate the use of tazarotene in photodamaged skin, Kang et al conducted a larger multicenter randomized double-blind vehicle-controlled parallel comparison of $0.01 \%, 0.025 \%, 0.05 \%$ and $0.1 \%$ tazarotene 
creams against $0.05 \%$ tretinoin emollient cream and vehicle cream applied once daily for 24 weeks (Kang et al 2001). Allergan Inc. funded the study of 349 volunteers aged 18 years or over with moderate facial photodamage as based on a 6-point scale at baseline using a photonumeric guideline ( 0 indicates no photodamage; 3 , moderate photodamage; 5 very severe photodamage). The topical treatments were applied once daily in the evening. Thirty- seven subjects did not complete the study-11 discontinued due to adverse events, however analysis was via intention to treat. Clinical outcome measures included overall assessment of photodamage (using the 6-point scale), global response to treatment (on a 7 point scale where 0 indicates complete response; 3 indicates moderate response and; 6 , condition worsened) and assessment of the following individual characteristics: fine wrinkling, mottled hyperpigmentation, lentigines, coarse wrinkling, tactile roughness, irregular depigmentation, pore size, elastosis, actinic keratoses, and telangiectasia. Subjects were also asked to perform a selfassessment of their response to treatment and whether they would continue to use the study medication. Histological features were assessed on samples taken from 31 subjects at baseline and week 24, and skin surface profilometry replicas of the crow's feet area were taken from 53 subjects ( 8 or 9 per group) pre- and post-treatment and assessed by computer assisted image analysis.

At week 24 the proportion of subjects with a significant improvement in fine wrinkling (at least 1 grade better compared with baseline) was significantly higher in all retinoid treatment groups, (apart from 0.025\% tazarotene which became significant at the week 26 post treatment evaluation) compared with vehicle $(\mathrm{p}<0.002)$. Amelioration of mottled hyperpigmentation compared to vehicle was only significant in the $0.05 \%$ tretinoin and $0.1 \%$ tazarotene cream groups. Overall global response to treatment (at least 1 grade improved from baseline) was 67\% with $0.1 \%$ tazarotene, $52 \%$ with $0.05 \%$ tazarotene, $41 \%$ with $0.01 \%$ tazarotene, $55 \%$ with $0.05 \%$ tretinoin and $22 \%$ with vehicle. Histological analysis post-treatment showed significant increased epidermal thickening compared with baseline in all retinoid groups, although this was not significant when compared with vehicle. All retinoids reduced melanin content but did not result in significant changes in cellular atypia, dermal elastosis, or type 1 collagen. Optical profilometry of skin surface replicas did not yield any significant results.

Treatment-related adverse events were generally mild to moderate, and included erythema, skin irritation and desquamation. Self-assessment showed $0.1 \%$ tazarotene and $0.05 \%$ tretinoin creams were favored over vehicle in evaluation of treatment response.

A further multicentre randomized study of the efficacy of once daily $0.1 \%$ tazarotene cream applied to the face compared with vehicle also confirmed the beneficial effects of tazarotene in treating the signs of photodamage (again funding for the study was provided by Allergan Inc) (Phillips et al 2002). The study consisted of a 24-week period where subjects were randomized to $0.1 \%$ tazarotene cream or vehicle followed by a 28 -week open-label extension where all subjects received tazarotene. Eighty six percent of the 563 subjects enrolled completed both phases of the trial with 20 of the 283 tazarotene treated patients discontinuing due to adverse effects in the double-blind phase. Subjects included were aged over 18 years, skin phototypes I, II, III or IV with at least mild severity of fine wrinkling and mottled hyperpigmentation and at least moderate severity of one of these. The main outcome measures were fine wrinkling and mottled hyperpigmentation and also included overall assessment of photodamage, global response to treatment and patient's assessment of photodamage. It is not stated whether analysis was based on intention to treat.

As in the previous study a 5-point scale was used to assess fine wrinkling, mottled hyperpigmentation, lentigines, elastosis, irregular pigmentation, tactile roughness, coarse wrinkling and telangiectasia, a 6-point scale was used to assess overall photodamage and a 7-point scale global improvement. Tazarotene resulted in a significantly greater incidence of clinical improvement (at least 1 grade) in both fine wrinkling (from week 8 onwards) and mottled hyperpigmentation (from week 2 onwards) compared with vehicle $(\mathrm{p}<0.01)$. There was also a significantly greater incidence of patients achieving improvement in lentigines, elastosis, pore size, coarse wrinkling, overall assessment of photodamage and global improvement in the tazarotene group. Telangiectasia and actinic keratoses were not significantly improved. More than $80 \%$ of the patients in the tazarotene group considered their photodamage at least somewhat improved from week 12 onwards compared with less than $45 \%$ of the patients using vehicle. During the open label phase there was continued clinical improvement in patients previously treated with tazarotene suggesting that in clinical practice prolonged treatment periods could yield enhanced results. The improvement noted in the vehicle treated group may have resulted partly from use of emollients and also from the requirement of patients to use a sunscreen and restrict sun exposure during the study. 
Monitoring of blood levels of tazarotenic acid in 48 patients who had received tazarotene in both phases of the trial showed that mean levels were $0.13 \mathrm{ng} / \mathrm{ml}$ : not exceeding $0.71 \mathrm{ng} / \mathrm{ml}$, and not increasing with duration of treatment (endogenous total plasma retinoic acid levels $6.6 \mathrm{ng} / \mathrm{ml}$ ). As expected, adverse events were mainly signs of skin irritation.

In order to further compare the effects of tazarotene and tretinoin in the treatment of photodamaged skin a multicenter, double blind, randomized parallel group study (supported by funding from Allergan Inc), evaluated tazarotene $0.1 \%$ cream and tretinoin $0.05 \%$ emollient cream used once daily for 24 weeks (Lowe et al 2004). Inclusion criteria and outcome measures were as for the previous study (inclusion criteria: at least mild fine wrinkling and mottled hyperpigmentation, and at least moderate levels of one of these). Subjects were also instructed to use sunscreen (=SPF 15) at least every morning, and analysis was on an intention to treat basis. One hundred and fifty-seven of 173 subjects enrolled completed the study: 3 subjects in the tazarotene group withdrew due to adverse events and 2 in the tretinoin group.

The incidence of treatment success, as defined by $50 \%$ or greater global improvement at the study endpoint, was $78 \%$ in the tazarotene group and $67 \%$ in the tretinoin group: statistical significance in favor of tazarotene was achieved at 16 weeks only. Other statistically significant differences achieved in favor of tazarotene included improvement in mottled hyperpigmentation at weeks 12 and 16 and overall assessment at week 16, although by week 24 improvement in fine wrinkling was the only variable with a statistically significant difference $(\mathrm{p}<0.01)$.

Eighty one percent of subjects in the tazarotene group and $84 \%$ in the tretinoin group reported they would continue to use their study medication if they chose to continue treatment for photodamage after the study. Adverse events were similar to previous studies; the only significant difference was a higher incidence of skin "burning" in the tazarotene group in the first week of treatment.

In 2005 a further randomized, double-blind trial of tazarotene $0.1 \%$ cream used for 24 weeks to treat facial photodamage was published (Kang, Krueger et al 2005). The purpose of this study was to confirm efficacy and tolerability of tazarotene. Inclusion criteria were as discussed previously. Primary outcome measures were again fine wrinkling and mottled hyperpigmentation as measured on the 5-point scale. Clinically significant improvement was defined as at least a $10 \%$ between group difference in the incidence of patients with at least a 1-grade improvement from baseline.
Secondary outcome measures included lentigines, irregular depigmentation, elastosis, apparent pore size, tactile roughness, coarse wrinkling, telangiectasia, overall integrated assessment of photodamage, and global response to treatment (as for the earlier study by Kang et al).

Photographs taken at baseline were used to aid investigators in evaluating response to treatment. Analysis for clinical efficacy was based on intention to treat.

Five hundred and five subjects, of 568 enrolled, completed the study: 14 discontinued due to adverse events. $63 \%$ of tazarotene treated subjects achieved clinical improvement in fine wrinkling at week 24 compared with $24 \%$ in the vehicle group $(\mathrm{p}<0.001)$ and $87 \%$ vs $43 \%$ achieved improvement in mottled hyperpigmentation $(\mathrm{p}<0.001)$. There were also significantly higher percentages of patients achieving clinical improvement in lentigines, irregular dyspigmentation, apparent pore size, elastosis, tactile roughness and overall integrated assessment. There were no significant between group differences in coarse wrinkling, telangiectasia or actinic keratoses. At week $24,93 \%$ of the tazarotene treated subjects considered their photodamage to be at least somewhat improved compared to $49 \%$ of vehicle treated subjects. Adverse events were more common in the active treatment group and included burning, desquamation and erythema. The authors comment that the reduction in apparent pore size may play an important role in overall patient satisfaction. Possible hypotheses to explain this reduction include retinoid-induced synthesis of collagen and/or a comedolytic affect.

\section{Histological response to tazarotene}

Machtinger et al conducted a double-blind randomized vehicle controlled trial to determine the histological effects of tazarotene $0.1 \%$ cream when applied to the face once daily for 24 weeks (Machtinger et al 2004). Inclusion criteria were the same as for previous studies. Three millimetre punch biopsies were taken from the crow's feet area at weeks 0 and 24. Primary outcome measures were improvement in keratinocytic and melanocytic atypia: overall assessments were made of cells that appeared abnormal in nuclear size, shape, staining characteristics or position relative to contiguous cells. Distribution of atypia was graded on a 4 point scale: 0 , not present; 1 , focal; 2 multifocal; and 3, diffuse. Assessment of epidermal and dermal parameters were also made, as were clinical efficacy measures as in the previous study.

Forty-eight of 50 patients randomized completed the study. Tazarotene treated patients were more likely to have 
either a reduction or no change in keratinocyte or melanocyte atypia compared with vehicle-treated patients (76\% vs 50\% and $80 \%$ vs $50 \%$ respectively), although the change in distribution or severity of keratinocytic atypia just failed to reach statistical significance $(p=0.055)$. Epidermal oedema, absent at baseline, increased significantly more with tazarotene than with vehicle $(\mathrm{p}<0.001)$. Epidermal thickness and epidermal polarity scores also increased significantly in the active treatment group $(\mathrm{p}=0.005$ and $\mathrm{p}=0.008$ respectively). Other measures including epidermal inflammation, abnormal elastin, number of epidermal melanocytes and dermal melanin did not show significant between group differences. The clinical efficacy of tazarotene was demonstrated by significant improvements (at least 1 grade improvement) in a higher percentage of patients than with the vehicle in overall assessment, mottled hyperpigmentation, irregular depigmentation, coarse wrinkling, elastosis and tactile roughness. No patients discontinued due to adverse events. The authors concluded that tazarotene $0.1 \%$ cream may help normalize cellular changes and thus reduce progression to precancerous states: perhaps by expression of the tumor suppressor gene, tazarotene inducible gene-3.

\section{Tazarotene in combination therapy}

Most recently Lowe et al have studied the efficacy of tazarotene $0.1 \%$ cream versus a combination of tazarotene $0.1 \%$ cream and hydroquinone $4 \%$ cream in the treatment of photodamaged facial skin (Lowe et al 2006). The trial was conducted as a double blind randomized study over a 24-week period. Inclusion criteria were similar to previous studies including mottled hyperpigmentation of at least moderate severity (on a 5 point scale) and an overall integrated assessment (of photodamage) score of at least moderate (on a 6 point scale). Patients were divided into two groups: tazarotene $0.1 \%$ cream in the evening and placebo cream each morning or tazarotene cream in the evening with hydroquinone $4 \%$ cream in the morning. Outcome measures included global response to treatment and changes in lentigines, mottled hyperpigmentation, irregular dyspigmentation, melasma, fine wrinkling, coarse wrinkling, elastosis, tactile roughness and overall integrated assessment.

One hundred and thirty-one subjects were enrolled in the study and 114 completed. Four subjects discontinued due to adverse events: two from the tazarotene alone group (hyperpigmentation and exacerbation of rosacea), and two from the tazarotene plus hydroquinone group (peeling, burning, and redness). Significantly more subjects from the tazarotene plus hydroquinone group achieved at least
$50 \%$ global improvement at weeks 8 to 16 , however by 24 weeks the results were comparable ( $88 \%$ with tazarotene plus hydroquinone versus $76 \%$ with tazarotene alone). The only other variables in which there were significant differences in the incidence of patients achieving at least a 1-grade improvement from baseline were lentigines $(76 \%$ in the tazarotene plus hydroquinone group, versus 54\% tazarotene alone at 24 weeks $\mathrm{p}<0.01)$ and mottled hyperpigmentation (92\% in the tazarotene plus hydroquinone group, versus $76 \%$ tazarotene alone at 16 weeks $\mathrm{p}<0.05$ ), although by 24 weeks there was no significant difference in improvement in mottled hyperpigmentation between the two groups. A higher proportion of patients reported complete improvement in photodamage with tazarotene plus hydroquinone ( $18 \%$ compared to $2 \%$ with tazarotene alone) and similar numbers reported at least definite improvement (75\% versus $72 \%$ ). The results from this study suggest that photodamaged patients with dyspigmentation may obtain greater clinical improvement with the use of hydroquinone in combination with the topical retinoid.

\section{Conclusion}

Available evidence indicates that in the short term at least topical tazarotene is safe and effective for the treatment of photodamaged skin. Furthermore, the clinical improvement seen in the tazarotene treated groups is consistent between the different trials. Adverse effects associated with tazarotene are mainly mild to moderate cutaneous irritation, which in clinical practice could perhaps be avoided by a more gradual institution of treatment.

The longest trial to date suggests that continued clinical improvement can occur beyond one year of treatment, and further trials to assess optimum duration of therapy and appropriate "maintenance" regimes to guide clinical practice are warranted.

\section{Summary points}

- Tazarotene is a synthetic retinoid selective for RAR $\beta$ and $\gamma$.

- There is good evidence from RCTs that tazarotene improves both the clinical and histological signs of photodamaged skin.

- Based on the available evidence tazarotene should be used once daily in conjunction with a sunscreen $(\mathrm{SPF}>15)$ for optimum results.

- Side effects of tazarotene tend to be mild, transient cutaneous irritation that may be reduced by a more gradual institution of treatment. 


\section{References}

Chandraratna RAS. 1996. Tazarotene-first of a new generation of receptorselective retinoids. British Journal of Dermatology, 135:18-25.

El-Domyati M, Attia S, Saleh F, et al. 2002. Intrinsic aging vs. photoaging: a comparative histopathological, immunohistochemical, and ultrastructural study of skin. Experimental Dermatology, 11:398-405.

Hadshiew IM, Eller MS, Gilchrest BA. 2000. Skin Aging and Photoaging: The Role of DNA Damage and Repair. American Journal of Contact Dermatitis, 11:19-25.

Kang S, Leyden J, Lowe NJ, et al. 2001. Tazarotene cream for the treatment of facial photodamage. Archives of Dermatology, 137:1597-604.

Kang S, Bergfield W, Gottlieb AB. 2005. Long-term efficacy and safety of tretinoin emollient cream $0.05 \%$ in the treatment of photodamaged facial skin. American Journal of Clinical Dermatology, 6:245-53.

Kang S, Krueger GG, Tanghetti EA, et al. 2005. A multicenter, randomised, double-blind trial of tazarotene $0.1 \%$ cream in the treatment of photodamage. Journal of the American Academy of Dermatology, 52:268-74.

Lowe NJ, Gifford M, Tanghetti E, et al. 2004. Tazarotene $0.1 \%$ cream versus tretinoin $0.05 \%$ emollient cream in the treatment of photodamaged facial skin: a multicenter, double-blind, randomised, parallel group study. Journal of Cosmetic and Laser Therapy, 6:79-85.
Lowe N, Horwitz S, Tanghetti E, et al. 2006. Tazarotene versus tazarotene plus hydroquinone in the treatment of photodamaged facial skin: A multicentre, double-blind, randomised study. Journal of Cosmetic Laser Therapy, 8:121-7.

Machtinger LA, Kaidbey K, Lim J, et al. 2004. Histological effects of tazarotene $0.1 \%$ cream vs. vehicle on photodamaged skin: a 6 month, multicentre, double-blind, randomised, vehicle controlled study in patients with photodamaged facial skin. British Journal of Dermatology, 151:1245-52.

Phillips TJ, Gottlieb AB, Leyden JL, et al. 2002. Efficacy of $0.1 \%$ tazarotene cream for the treatment of photodamage. Archives of Dermatology, 138:1486-93.

Samuel M, Brooke RC, Hollis S, et al. 2005. Interventions for photodamaged skin. Cochrane Database of Systematic Reviews, CD001782.

Sefton J, Kligman AM, Kopper SC, et al. 2000. Photodamage pilot study: A double-blind, vehicle-controlled study to assess the efficacy and safety of tazarotene $0.1 \%$ gel. Journal of the American Academy of Dermatology, 43:656-63.

Yaar M, Gilchrest B. 1990. Cellular and molecular mechanisms of cutaneous aging. Journal of Dermatologic Surgery and Oncology, 16:915-22. 